\title{
Coordinación de política económica mediante el estudio de la relación Dinámica-Macroeconómica de la cartera de morosidad de consumo del Ecuador en el periodo 2009-2019
}

\section{Economic policy coordination through the study of the Macroeconomic-Dynamics relationship of the Ecuadorian consumer delinquency portfolio in the period 2009-2019}

\author{
Luis Eduardo Peñafiel Chang \\ Pontificia Universidad Católica Argentina \\ Buenos Aires, Argentina \\ lueduardo1994@gmail.com \\ Orcid: 0000-0002-5571-3978
}

\author{
Gisella Beatriz Camelli \\ Redmas Arg - Grupo Cisnero \\ Buenos Aires, Argentina \\ gbcamelli@gmail.com \\ Orcid: 0000-0002-6204-5824
}

\begin{abstract}
Resumen - Este estudio busca examinar el comportamiento y efectos de los componentes que determinan la dinámica de la morosidad de consumo del Ecuador durante 2009-2019, periodo comprendido con valores mensuales. Se utiliza una de las alternativas más apropiadas para su modelización en la formación del modelo macroeconómico: la técnica econométrica de los vectores autorregresivos (VAR) y el proceso autorregresivo integrado de promedios móviles (ARIMA). Se estudia su definición y se deduce cómo obtener su estimación. Se revela que paradójicamente contrario a lo encontrado en la literatura, la liquidez total $\left(M_{2}\right)$ no soporta atribuciones significativas en la calidad de la cartera de morosidad de consumo, mientras que un choque de desviación estándar al tipo de cambio real en el primer periodo no tiene un impacto representativo, sin embargo, a posteriori mejora notablemente la calidad de cartera. Se comprueba empíricamente que la morosidad de consumo tiene memoria y se enfatiza el gran desafío para la coordinación de la política económica y mantener en constante crecimiento la actividad económica (PIB), dado que esta medida afecta significativamente el nivel de morosidad de consumo.
\end{abstract}

Palabras Clave: Modelo Estructural Dinámico, Proceso autorregresivo, Series de tiempo, Crecimiento económico.

\begin{abstract}
This study seeks to examine the behavior and effects of the components that determine the dynamics of nonperforming loans in Ecuador during the 2009-2019 period, comprised of monthly values. One of the most appropriate alternatives for its modeling in the formation of the macroeconomic model is employed: the econometric technique of autoregressive vectors (VAR) and the integrated autoregressive process of moving averages (ARIMA). Its definition is studied, and the way to obtain its estimate is deduced. It is found that paradoxically contrary to what is stated in the literature, total liquidity (M 2) does not support significant attributions in the quality of the portfolio of non-performing loans, while a standard deviation shock to the real exchange rate in the first period does not have a representative shock, however, a posteriori, it improves the portfolio quality considerably. It is empirically verified that the delinquency of consumption has memory and the great challenge for the coordination of economic policy is emphasized to keep the economic activity (GDP) in constant growth, since this measure significantly affects the level of delinquency of consumption.
\end{abstract}

Keywords: Dynamic structural model, Autoregressive process, Time series, Economic growth.

Classification JEL: C12; C32; C52; E52; G19

Sumario: I Introducción, II Desarrollo de contenidos, III Metodología, IV Resultados, V Conclusiones.

Como citar: Peñafiel Chang, Luis. (2020). Coordinación de política económica mediante el estudio de la relación Dinámica Macroeconómica de la cartera de impago de consumo del Ecuador periodo (2009-2019). Revista Tecnológica - Espol, $32(1)$. Recuperado http://www.rte.espol.edu.ec/index.php/tecnologica/article/view/722 


\section{INTRODUCCIÓN}

Este trabajo es una extensión del artículo de [1] en el que se presenta una forma alternativa de medir la dinámica de la Morosidad del Sistema Financiero en Ecuador. El punto de partida de estos modelos se encuentra en el trabajo del Fondo Monetario Internacional que desarrolló un modelo VAR para Argentina como parte de pruebas de estrés considerando series trimestrales durante el periodo de 1993-2012 siendo el modelo,

$$
D_{0} Y_{t}=\mathrm{j}+D_{1} Y_{t-1}+\ldots+D_{p} Y_{t-p}+u_{t}
$$

Donde el vector de variables endógenas es representado como $Y_{t}$ que contiene el crecimiento económico de Brasil y Estados Unidos, el índice VIX de Estados Unidos, la tasa de interés de Estados Unidos, la tasa de interés interna y el tipo de cambio efectivo nominal. La letra $\mathrm{j}$ representa un vector de constantes, D una matriz $n x n$ de coeficientes y $u_{t}$ el vector de shocks estructurales que es ruido blanco. El crecimiento de los socios comerciales es exógeno a todas las demás variables del sistema son los supuestos asociados al modelo, los shocks de aversión al riesgo global responden a shocks de crecimientos de los socios, la tasa de Estados Unidos responde al PIB de Estados Unidos, los shocks de términos de intercambio responden de forma contemporánea al crecimiento de los socios comerciales [2].

La bibliografía consultada para la realización de este trabajo incluye, entre otros títulos, económicos [3], [4], [5], [6], [7], [8], [9], [10], [11] [12], [13], [14] quienes de manera sintética presentan prototipos de modelos para determinar la interrelación de las variables en el tiempo teniendo en cuenta que cambios en las circunstancias económicas tienen efectos perceptibles en las tasas de impago de los créditos. El Banco Asiático de Desarrollo también realiza un aporte destacado al describir conceptos y herramientas de evaluación de la firmeza de los Sistemas Financieros para las economías de Asia, América del Sur y Europa [15].

Dado este contexto, el presente documento se divide en cinco apartados que incluyen esta introducción. En el segundo apartado se revelará la literatura referente al tema revisando los modelos construidos a una categoría internacional y nacional. En el tercer apartado se hace un cotejo del modelo macroeconómico describiendo la herramienta teórica de una de las alternativas más apropiadas para su modelización: la técnica econométrica de los vectores autorregresivos (VAR) y el proceso autorregresivo integrado de promedios móviles (ARIMA). En el cuarto apartado se presentan los resultados obtenidos y, por último, el quinto apartado contiene las conclusiones de la presente investigación.

\section{DESARROLLO DE CONTENIDOS}

Una de las alternativas para estimaciones de modelos macroeconómicos además del (VAR) son los de equilibrio general dinámico y estocástico (DSGE) y los modelos de estadística pura.

Los modelos de equilibrio general dinámico y estocástico (DSGE) son los de menor simplicidad para describir el comportamiento de un gran agregado de agentes económicos tales como empresas, familias, gobierno, sector financiero entre otros. Además, suponen que los agentes toman sus decisiones de carácter individual y de esta manera maximizan sus funciones de utilidad, la interacción entre si a través del mercado hace que estos modelos tengan una gran cuantía de parámetros de comportamiento y se los utilice asiduamente para investigaciones de impacto de políticas económicas [16].

En Ecuador [17] se propuso un modelo (DSGE) en el que se explica las variables macroeconómicas reales como: PIB, Consumo, Inversión, mercado de trabajo y el mercado monetario. Por su parte, [18] se estructura un modelo VAR para Ecuador con el fin de determinar el comportamiento de la cartera comercial con series mensuales en el periodo 2007-2015 siendo el modelo:

$$
F_{t}=a_{1} F_{t-1}+a_{2} F_{t-2}+\ldots .+a_{p} F_{t-p}+\mathrm{b} X_{t}+e_{t}
$$

Donde las variables endógenas contenidas en $F_{t}$ son: La cartera Improductiva Comercial, Tasa de Morosidad comercial y Crecimiento de la cartera total. El vector de variables exógenas $X_{t}$ incluye la variación anual del PIB real, Índice de precios al consumidor, tasa de Desempleo, Riesgo País, variación deuda Pública y Liquidez del Sistema Financiero.

Por otro lado, [12] analizando la categoría internacional estudia el choque del comportamiento macroeconómico relacionado con la morosidad de la cartera de consumo en Colombia con periodicidad trimestral comprendida entre el 2002 al 2015 siendo el modelo:

$$
V_{t}=b_{1} V_{t-1}+B_{2} V_{t-2}+\ldots+b_{p} V_{t-p}+e_{t}
$$

Donde las variables contenidas en el vector $\mathrm{V}$ fueron en orden descendente de la más exógena a la más endógena las cuales se tiene: la calidad de la cartera de consumo, el crecimiento del PIB real, la inflación anual y la tasa de interés DTF.

En otro orden de ideas, la vinculación de las variables macroeconómicas es permitido mediante un estudio de cópulas de los modelos de estadística pura, esta herramienta ha sido desarrollada en los últimos años y es una técnica que viene despertando un progresivo interés como el caso de la serie de documentos denominados Systemic Risk Monitor publicados por el Banco Central de Austria [19].

La utilización reducida de la teoría económica para abogar la vinculación entre las variables a manejarse en los modelos VAR que distingue a los modelos de ecuaciones simultáneas [20]. No es simple conseguir conclusiones refrentes al recorrido de la causalidad de una variable mientras los VAR se focalicen en el diagnostico dinámico $\mathrm{y}$ en resultante sobre la diferenciación entre variables endógenas y exógenas. Dicho de otro modo, permitir realzar ensayos de comportamientos dinámicos y elaborar proyecciones, hace que sean herramientas útiles si se determinan simultáneamente [21]. 


\section{METODOLOGÍA}

Se consideró un modelo de Vectores Autorregresivos (VAR) con el objetivo de examinar la interacción auténtica entre las variables macroeconómicas y la calidad de la cartera del consumo para el periodo comprendido entre 2009-2019. El cual para distinguir las relaciones de carácter simultánea y exponer los comportamientos más probables frente a la materialización de un choque en una variable se permitió trabajar con series de tiempo multivariadas. Conjuntamente, este instrumento ayuda a modelar este tipo de series cuidando que los cambios en las circunstancias económicas no suelen mantener un efecto inmediato en la tasa de impago de consumo [22]. Por otro lado, también se realiza un modelo estocástico (ARIMA) para identificar la existencia de memoria en la morosidad de consumo.

Un sistema de ecuaciones aparentemente no relacionadas cuya estimación puede realizarse por mínimos cuadrados clásicos (MCC) es un modelo de vectores autorregresivos (VAR) [23]. Un sistema VAR de dos ecuaciones endógenas siguiendo la metodología propuesta por [24] y [1] tiene la forma:

$$
\begin{aligned}
& X_{t}=b_{10}-b_{12} W_{t}+\gamma_{11} \mathrm{X}_{t-1} \gamma_{12} \mathrm{~W}_{t-1}+\epsilon_{x t} \\
& W_{t}=b_{20}-b_{21} X_{t}+\gamma_{21} \mathrm{X}_{t-1}+\gamma_{22} \mathrm{~W}_{t-1}+\epsilon_{w t}
\end{aligned}
$$

Donde $X_{t}$ y $W_{t}$ son las variables endógenas. Las mismas deben tener media, varianza constante y función de autocorrelación finita, es decir, deben ser estacionarias. Las perturbaciones $\epsilon_{x t}$ y $\epsilon_{w t}$ deben ser ruido blanco, procesos con media cero, desvío estándar constante $\left(\sigma_{x} \mathrm{y}\right.$ $\left.\sigma_{w}\right)$ y sin autocorrelación.

Los parámetros reflejan la vinculación entre las variables endógenas, siendo, por ejemplo, $-b_{12}$ consecuencia contemporánea de una alteración en una unidad en $\mathrm{W}_{t}$ sobre $\mathrm{X}_{t}, \mathrm{o} \gamma_{12}$ el resultado de un cambio en una unidad en $\mathrm{W}_{t-1}$ sobre $X_{t}$. De igual modo, la estipulación $-b_{21} \neq 0$ conlleva a $\epsilon_{x t}$ que tenga efecto indirecto sobre $W_{t}$ y equivalentemente $-b_{12} \neq 0$ repercute que $\epsilon_{w t}$ tenga efecto indirecto sobre Xt.

Debido a la presencia de paralelismo entre $X_{t}, W_{t}$ y dado que coexiste correlación entre $X_{t} \mathrm{y} \epsilon_{w t}\left(\mathrm{y}\right.$ entre $\left.W_{t}, \epsilon_{t}\right)$, quebrantando el supuesto de exogeneidad de los regresores no es factible estimar por mínimos cuadrados clásicos cada ecuación por emancipado, sino que deben estimarse los parámetros del sistema en forma conglomerada. Para eso, es indispensable manifestar el sistema en su forma reducida. Iniciar el sistema estructural apuntado en forma matricial es:

$$
\left[\begin{array}{cc}
1 & b_{12} \\
b_{21} & 1
\end{array}\right]\left[\begin{array}{l}
X_{t} \\
W_{t}
\end{array}\right]=\left[\begin{array}{l}
b_{10} \\
b_{20}
\end{array}\right]+\left[\begin{array}{ll}
\gamma_{11} & \gamma_{12} \\
\gamma_{21} & \gamma_{22}
\end{array}\right]\left[\begin{array}{l}
X_{t-1} \\
W_{t-1}
\end{array}\right]+\left[\begin{array}{c}
\epsilon_{x t} \\
\epsilon_{w t}
\end{array}\right]
$$

$$
Z_{t} B=\pi_{0}+\pi_{1} Z_{t-1}+e t
$$

$$
\begin{aligned}
& B=\left[\begin{array}{cc}
1 & b_{12} \\
b_{21} & 1
\end{array}\right] \quad Z_{t}=\left[\begin{array}{l}
X_{t} \\
W_{t}
\end{array}\right] \quad \pi_{0}=\left[\begin{array}{l}
b_{10} \\
b_{20}
\end{array}\right] \quad \pi_{1} \\
= & {\left[\begin{array}{ll}
\gamma_{11} & \gamma_{12} \\
\gamma_{21} & \gamma_{22}
\end{array}\right] \quad \mathrm{e}_{t}=\left[\begin{array}{c}
\epsilon_{x t} \\
\epsilon_{w t}
\end{array}\right] }
\end{aligned}
$$

Multiplicando por $B-1$, se llega a la forma reducida:

$$
\begin{gathered}
\mathrm{Z}_{t}=A_{0}+A_{1} Z_{t-1}+e_{t} \\
A_{0}=B^{-1} \pi_{0} \\
\text { et }=B^{-1} \epsilon_{t}
\end{gathered}
$$

Escrito en forma vectorial es:

$$
\begin{aligned}
& X_{t}=a_{10}-a_{11} X_{t-1}+a_{12} W_{t-1}+e_{1 t} \\
& W_{t}=a_{20}+a_{21} X_{t-1}+a_{22} W_{t-1}+e_{2 t}
\end{aligned}
$$

Colisionadas por los errores de las dos ecuaciones del sistema primitivo están las perturbaciones del sistema reducido:

$$
\begin{aligned}
& e_{1 t}=\frac{(11)}{\epsilon_{x t}-b_{12} \epsilon_{w t}} \\
& 1-b_{12} b_{21} \\
& e_{2 t}=\frac{(12)}{\frac{\epsilon_{x t}-b_{21} \epsilon_{x t}}{1-b_{12} b_{21}}}
\end{aligned}
$$

El uno y el otro son procesos estacionarios, por efectuar las propiedades de la definición de ruido blanco, es decir tienen: 1) media cero, 2) varianza constante, 3) La autocovarianza es independiente del tiempo igual a cero. Consecuentemente, se define la matriz de varianzas y covarianzas como:

$$
\begin{gathered}
\operatorname{Var} \operatorname{Cov}=\left[\begin{array}{cc}
\operatorname{var}\left(e_{1 t}\right) & \operatorname{cov}\left(e_{1 t}, e_{2 t}\right) \\
\operatorname{cov}\left(e_{1 t}, e_{2 t}\right) & \operatorname{var}\left(e_{2 t}\right)
\end{array}\right]= \\
{\left[\begin{array}{cc}
\sigma_{1}^{2} & \sigma_{12} \\
\sigma_{21} & \sigma_{2}^{2}
\end{array}\right]}
\end{gathered}
$$

Especificación del VAR implica la denominación del conjunto de parámetros que escoltarán la estructura autorregresiva. Dos normas comúnmente usadas es el de información de Akaike (AIC) y el criterio de información del error de predicción final (FPE)

$$
A I C=-2\left(\frac{L L}{T}\right)+\left(\frac{2 t_{p}}{T}\right)
$$

Donde $T$ es el número de observaciones, $t_{p}$ es el total de parámetros del modelo y $L L$ el log likelihood.

$$
\mathrm{FPE}=\sum_{u}\left(\frac{T+\overline{\mathrm{m}}}{T-\overline{\mathrm{m}}}\right) \wedge k
$$

Siendo $K$ la cantidad de ecuaciones y $\bar{m}$ el número promedio de parámetros en las $K$ ecuaciones.

Inspeccionando el modelo de dos ecuaciones, los seis parámetros $a_{10}, a_{20}, a_{11}, a_{21}, a_{12}, a_{22}$ y las varianzas y 
covarianzas de los errores $\operatorname{var}\left(e_{1}\right), \operatorname{var}\left(e_{2}\right)$ y $\operatorname{cov}\left(e_{1} ; e_{2}\right)$ son posibles de estimar, a pesar de ello los diez parámetros del VAR estructural no serán posibles estimar, esto quiere decir, que al menos se debe imponer alguna restricción sobre el modelo. Por esta razón [23] plantea como restricción $b_{21}=0$ adicionando asimetría al modelo. Por lo cual, los errores del sistema reducido pasan a ser:

$$
\begin{gathered}
e_{1 t}=\epsilon_{x t}-b_{12} \epsilon_{w t} \\
e_{2 t}=\epsilon_{w t}
\end{gathered}
$$

Esto conlleva que $X_{t}$ no tiene choque en $W_{t}$. De igual forma, un shock por medio de $\epsilon_{x t} \mathrm{y} \epsilon_{w t}$ afecta $X_{t}$ pero solo uno en $\epsilon_{w t}$ colisiona en $W_{t}$. Esto da como consecuencia que la matriz de varianzas y covarianzas de los errores pasen a conformar un sistema de tres ecuaciones donde las incógnitas son $\sigma_{x}^{2}, \sigma_{w}^{2}, \mathrm{~b}_{12}^{2}$. A esto se lo conoce como descomposición de Cholesky. Reflejado matricialmente se aprecia como despejar dichas variables. Dado el sistema VAR de la ecuación (7) multiplicado por $B^{-1}$ tenemos:

$$
\begin{aligned}
& {\left[\begin{array}{l}
X_{t} \\
W_{t}
\end{array}\right]=\left[\begin{array}{cc}
1 & -b_{12} \\
0 & 1
\end{array}\right]\left[\begin{array}{l}
b_{10} \\
b_{20}
\end{array}\right]+\left[\begin{array}{cc}
1 & -b_{12} \\
0 & 1
\end{array}\right]\left[\begin{array}{ll}
\gamma_{11} & \gamma_{12} \\
\gamma_{21} & \gamma_{22}
\end{array}\right]} \\
& {\left[\begin{array}{l}
X_{t-1} \\
W_{t-1}
\end{array}\right]+\left[\begin{array}{cc}
1 & -b_{12} \\
0 & 1
\end{array}\right]\left[\begin{array}{c}
\epsilon_{x t} \\
\epsilon_{w t}
\end{array}\right]} \\
& {\left[\begin{array}{l}
X_{t} \\
W_{t}
\end{array}\right]=\left[\begin{array}{c}
b_{10}-b_{12} b_{10} \\
b_{20}
\end{array}\right]+} \\
& {\left[\begin{array}{cc}
\gamma_{11}-b_{12} \gamma_{21} & \gamma_{12}-b_{12} \gamma_{22} \\
\gamma_{21} & \gamma_{22}
\end{array}\right]\left[\begin{array}{l}
X_{t-1} \\
W_{t-1}
\end{array}\right]+\left[\begin{array}{c}
\epsilon_{x t}-b_{12} \epsilon_{w t} \\
\epsilon_{w t}
\end{array}\right]}
\end{aligned}
$$

Entonces igualando cada ecuación a las obtenidas en (9) y (10) se logran las siguientes seis ecuaciones:

$$
\begin{gathered}
a_{10}=b_{10}-b_{12} b_{20} \\
a_{20}=b_{20} \\
a_{12}=\gamma_{12}-b_{12} \gamma_{22} \\
a_{11}=\gamma_{11}-b_{12} \gamma_{21} \\
a_{21}=\gamma_{21} \\
a_{21}=\gamma_{21}
\end{gathered}
$$

Engendrar un sistema de nueve ecuaciones con incógnitas que se encuentre identificado se logra integrando los tres componentes $\operatorname{var}\left(e_{1}\right), \operatorname{var}\left(e_{2}\right) y$ $\operatorname{cov}\left(e_{1} ; e_{2}\right)$. Un VAR con $\mathrm{n}$ variables en términos global donde $\mathrm{B}$ es una matriz de $\mathrm{n} * \mathrm{n}$ presenta $\mathrm{n}$ regresiones para los residuos y $\mathrm{n}$ regresiones para los shocks estructurales y para que el modelo este identificado es indispensable que $\frac{\left(n^{2}-n\right)}{2}$ sea igual a cero. Esto se consigue mediante la descomposición de Cholesky donde a la matriz B la convierte triangular.

\subsection{Definición del VAR para la morosidad de consumo del Ecuador.}

El periodo 2009-2019 fue cubierto para desarrollar el modelo de vectores autorregresivos (VAR) para la morosidad de consumo del Ecuador. Se realizaron pruebas con un conjunto acumulado de variables que han sido utilizadas en otros trabajos recientes como se menciona en la indagación bibliográfica.

El modelo queda desarrollado por cuatro variables endógenas y una exógena al igual que el trabajo de [1] dado que fueron las más significativas para la morosidad de consumo del Ecuador. Las variables se miden en variaciones interanuales y fueron obtenidas del Banco Central del Ecuador (BCE). Este estudio tiene periodicidad mensual lo que permite amplificar la cantidad de observaciones para el periodo seleccionado añadiendo solidez al modelo. Se consideraron las entidades financieras que se encuentran operativas en la última fecha disponible para el índice de impago de consumo del Ecuador.

La segunda variable endógena es la liquidez total o dinero que en sentido amplio incluye la oferta monetaria y el cuasidinero. Por otro lado, dado que el BCE publica la serie Producto Interno Bruto (PIB) con frecuencia trimestral esta fue la única que debió ser adaptada, en su sustitución se utilizó una variable que mide el nivel de actividad (IDEAC) al ser un proxy del cotejo trimestral que en adelante será llamada PIB y es elaborado en base a ponderaciones sectoriales de diversas actividades del sector real, el cual mide el avance económico coyuntural del país.

La cuarta variable es el tipo de cambio real multilateral, indicador de la competitividad de la economía, se estableció a partir del tipo de cambio nominal (TCN) deflactado por el índice de precios de consumo (IPC) ambos elaborados por el BCE. Otra variable considerada como posible endógena, pero luego dejada de lado, fue el total de la cartera de crédito desechada por no presentar la influencia sobre el nivel de actividad y la liquidez que se esperaba. Del mismo modo entre los agregados se probaron resultados con las variables $M_{1}$ y el coeficiente entre préstamos y depósitos ambos del sector financiero, pero fueron relegadas a favor de $M_{2}$.

Un factor externo con probada atribución en la economía ecuatoriana, dado su colisión en el efecto de la cuenta corriente mediante las exportaciones, es el precio de petróleo el cual fue seleccionado como variable exógena, esta serie es publicada por el (BCE). Otros indicadores externos conexos con el impacto en el sistema financiero y económico fue el indicador de reservas internacionales descartándose porque se observó que la variable es muy dependiente de la circunstancia política lo que vicia el análisis.

En las ecuaciones (26) a (29) se evidencia como queda construido el modelo reducido donde $M_{o c t}$ es la morosidad de consumo, $M_{2 t}$ la liquidez del sistema financiero, $Y_{t}$ el PIB, el tipo de Cambio Real representado como TCR y el precio del petróleo como $S_{t}$.

$$
\begin{gathered}
M_{o c t}=u_{10}+u_{11} M_{o t-1}+u_{12} M_{2 t-1}+u_{13} Y_{t-1}+u_{14} T C R_{t-1}+ \\
u_{15} S_{t-1}+e_{1 t}(26)
\end{gathered}
$$




$$
\begin{aligned}
& M_{2 t}=u_{17}+u_{18} M_{o t-1}+u_{19} M_{2 t-1}+u_{20} Y_{t-1}+u_{21} T C R_{t-1}+ \\
& u_{22} S_{t-1}+e_{2 t} \quad(27) \\
& Y_{t}=u_{24}+u_{25} M_{o t-1}+u_{26} M_{2 t-1}+u_{27} Y_{t-1}+u_{28} T C R_{t-1}+ \\
& u_{29} S_{t-1}+e_{3 t} \\
& T C R_{t}=u_{30}+u_{31} M_{o t-1}+u_{32} M_{2 t-1}+u_{33} Y_{t-1}+u_{34} T C R_{t-1} \\
& +u_{35} S_{t-1}+e_{4 t}(29)
\end{aligned}
$$

Y expresado matricialmente,

$$
\begin{aligned}
{\left[\begin{array}{c}
M_{o c t} \\
M_{2} \\
Y_{t} \\
T C R
\end{array}\right] } & {\left[\begin{array}{l}
u_{10} \\
u_{17} \\
u_{24} \\
u_{30}
\end{array}\right]\left[\begin{array}{llll}
u_{11} & u_{12} & u_{13} & u_{14} \\
u_{18} & u_{19} & u_{20} & u_{21} \\
u_{25} & u_{26} & u_{27} & u_{28} \\
u_{31} & u_{32} & u_{33} & u_{34}
\end{array}\right]\left[\begin{array}{c}
M o_{t-1} \\
M 2_{t-1} \\
Y t_{t-1} \\
T C R_{t-1}
\end{array}\right] } \\
+ & {\left[\begin{array}{l}
u_{15} \\
u_{22} \\
u_{29} \\
u_{45}
\end{array}\right][S t]+\left[\begin{array}{l}
e_{1 t} \\
e_{2 t} \\
e_{3 t} \\
e_{4 t}
\end{array}\right] }
\end{aligned}
$$

También se consideró el proceso autorregresivo integrado de promedios móviles (ARIMA). Introducida originariamente a través de los trabajos pioneros de Box y Jenkins, los modelos autorregresivos y de promedios móviles (ARMA) publicación que probó ser bastante efectiva a pesar de su sencilla sistematización, el cual, se instauró de esta manera como una solución relevante a los modelos estructurales, entonces muy extendidos en experiencia de predicciones [25].

La esencial distinción entre los modelos ARMA y los clásicos es el punto de vista estocástico que se le da a las series de tiempo, en lugar de tratarlas de carácter determinista. Desde este punto de vista se concibe a la serie de tiempo como un conjunto de valores de tipo aleatorio, generados desde un proceso completamente desconocido, dicho de otra forma, se percibe a la serie como un proceso estocástico. Del mismo modo, derivado del desconocimiento del proceso causante de los datos, el propósito de este enfoque es tratar de determinar el modelo probabilístico que reproduzca las características principales del comportamiento de la serie [26].

El procedimiento acostumbrado de plasmar y emplear modelos ARIMA es utilizando la metodología elaborada por Box y Jenkins. Si bien existen diferentes variantes de cómo esta se aplica, la que divide el proceso en cuatro pasos es la forma más habitual: identificación, estimación del modelo, diagnóstico y predicción. En esta sección, se seguirá esta estructura a excepción de la predicción porque solo se busca identificar la existencia de memoria en las series.

La identificación empieza al establecer el orden de diferenciación $\mathrm{d}$, se tiene la transformación estacionaria de la serie $Z_{t}=(1-\mathrm{L})^{d} Y_{t}$ que se puede figurar mediante un proceso $\operatorname{ARMA}(\mathrm{p}, \mathrm{q})$ estacionario.

En este estado se pretende identificar los órdenes p y q del proceso que puede reproducir las características de la serie estacionaria y de analizar la conveniencia de la incorporación del parámetro asociado a la media. Las propiedades de la serie estacionaria se analizan en el capítulo cuatro.

La naturaleza dinámica del proceso estacionario está recogida en la función de autocorrelación FAC por lo que ésta será el artilugio elemental para identificar los órdenes p y q del modelo ARMA apropiado para suplir las características de la serie estacionaria $Z_{t}$. Los coeficientes de autocorrelación muestral de $Z_{t}$ son:

$$
p_{k}=\rho Y_{t} Y_{t-k}, Y_{t-1}, Y_{t-2}, \ldots, Y_{t-k+1}
$$

Por consiguiente, el coeficiente de autocorrelación parcial $p_{k}$ es el coeficiente de la subsecuente regresión lineal.

$$
Y_{t}=\alpha+p_{1} Y_{t-1}+p_{2} Y_{t-2}+\ldots+p_{k} Y_{t-k}+e_{t}
$$

Las propiedades de la función de autocorrelación parcial (FACP) son análogas a las de la función de autocorrelación (FAC), dicho de otro modo, los coeficientes $p_{k}$ no se sujetan de unidades y son inferiores que la unidad en valor absoluto, $p_{0}=1$ y $p_{1}=\rho_{1}$, la FACP es una función simétrica, la FACP de un proceso estocástico estacionario decrece prontamente hacia cero cuando $\mathrm{k} \rightarrow \infty$.

Estimar a partir de los datos de la serie como una función de los coeficientes de autocorrelación simples estimados $\widehat{\rho_{k}}$, se logra la función de autocorrelación parcial FACP. La conformación de la función de autocorrelación parcial para un modelo estacionario $\operatorname{ARMA}(p, q)$ es como sigue:

$$
\begin{aligned}
& Y_{t}=\alpha_{t}+p_{1} Y_{t-1}+p_{2} Y_{t-2}+\ldots+p_{p} Y_{t-p}+\alpha_{t} \phi 1 \alpha_{t-1} \\
& +\phi 2 \alpha_{t-2}+\cdots+\phi q \alpha_{t-q}
\end{aligned}
$$

La FACP de un modelo ARMA (p, q) es infinita y los p primeros coeficientes de la FACP dependerán de los parámetros autorregresivos y de los de medias móviles y desde el inicio del retardo $\mathrm{p}+1$ consiste solamente de la estructura de la parte de medias móviles, de manera que, decrece prontamente hacia cero de manera exponencial cuando las raíces del polinomio de medias móviles son reales y en forma de onda seno-coseno suavizada si las raíces del citado polinomio son complejas.

Contrastando la complexión de las funciones de autocorrelación simple y parcial estimadas con las características básicas de las funciones de autocorrelación teóricas de la TABLA I se puede identificar los procesos que podrían haber producido la serie bajo estudio.

Análogamente, para la identificación del modelo adecuado solo se alcanza con las estimaciones de la FAC y de la FACP a partir de los datos de la serie. Los estimadores de los coeficientes de autocorrelación son variables aleatorias que tiene una distribución de probabilidad y captará valores para diferentes realizaciones $\operatorname{de} Y_{t}$. De tal forma que, para identificar el orden del proceso ARMA ( $p$, q) apropiado para la serie, es imprescindible precisar la estructura de las series FAC y FACP estimadas realizando contrastes sobre la significación individual de los coeficientes de autocorrelación simple y parcial estimados. 
TABLA I

CRITERIOS PARA DETERMINAR EL VALOR DE LOS PARÁMETROS PARA UN ARMA $(\mathrm{P}, \mathrm{Q})$. FAC FACP

MA(q) $\quad \begin{array}{ll}\text { Picos } & \text { Decrece } \\ & \text { significativos en } \\ & \text { exponencialmente }\end{array}$

$\begin{array}{lll}\text { AR(p) } & \begin{array}{l}\text { Disminuye } \\ \text { exponencialmente } \\ \text { o con un patrón de } \\ \text { onda sinusoide } \\ \text { achatada o ambos }\end{array} & \begin{array}{l}\text { Picos } \\ \text { significativos en } \\ \text { los rezagos q }\end{array} \\ \text { ARMA }(\mathrm{p}, \mathrm{q}) & \begin{array}{l}\text { Decrece } \\ \text { exponencialmente }\end{array} & \begin{array}{l}\text { Decrece } \\ \text { exponencialmente }\end{array}\end{array}$

Fuente: [21]

Por otra parte, hay que tener en cuenta que no es una tarea sencilla identificar el modelo a través de las funciones de autocorrelación simple y parcial estimadas cuando se procede con la identificación de un modelo $\operatorname{ARMA~(p,~q).~}$ En esta primera etapa no se trata tanto de identificar el modelo correcto, sino de acotar un subconjunto de modelos ARIMA que han podido generar la serie. Posteriormente, en la etapa de estimación y validación, dependiendo de los resultados, se vuelve a esbozar la identificación del proceso.

Generalmente, se trata de buscar los modelos más simples que reproduzcan las características de la serie. Es preciso tener en consideración que, por un lado, hay cierta probabilidad de conseguir algún coeficiente significativo, pese a que los datos fueran generados por un ruido blanco y en otro orden de cosas que al determinar qué retardos pueden ser significativamente distintos de cero, del mismo modo, hay que considerar la interpretación que se les pueda dar. Asimismo, los coeficientes de autocorrelación muestral están correlacionados entre sí, de modo que en el correlograma muestral pueden presentarse ciertas ondulaciones que no se corresponden con el correlograma teórico. El ejercicio de identificación será más fácil cuanto más grande sea el tamaño de la muestra.

Después de que se han identificado los procesos estocásticos que han podido generar la serie temporal $Y_{t}$, la siguiente fase depende en estimar los parámetros desconocidos de dichos modelos.

$$
\mathrm{B}=\left(\alpha, p_{1}, \ldots, p_{p}, \phi_{1}, \ldots, \phi_{\mathrm{p}}\right) \quad ; \mathrm{y} \quad \sigma_{a}^{2}
$$

Estos parámetros se pueden estimar de forma consistente por Mínimos Cuadrados o Máxima Verosimilitud. Los dos métodos de estimación se basan en el cómputo de las innovaciones $\alpha_{t}$, a partir de los valores de la serie estacionaria. El método de Mínimos Cuadrados minimiza la suma de cuadrados.

$$
\operatorname{Min} \sum_{t}^{\infty} \mathrm{a}_{t}^{2}
$$

La función de verosimilitud se puede derivar a partir de la función de densidad conjunta de las innovaciones, $a_{1}$, $a_{2}, \ldots, a_{\mathrm{t}}$ que, bajo supuesto de normalidad, es como sigue:

$$
f\left(a_{1}, a_{2}, \ldots, a_{\mathrm{t}}\right) \mid \infty \sigma^{T} \exp \left\{-\sum_{t=1}^{T} \frac{\mathrm{a}_{t}^{2}}{2 \sigma^{2}}\right\}
$$

Para solucionar el problema de estimación, las ecuaciones (35) y (36) se han de denotar en función del conjunto de información y de los parámetros desconocidos del modelo. Para un modelo ARMA (p, q) la innovación se puede escribir de acuerdo con:

$$
\left(a_{\mathrm{t}}=Z_{t}-\alpha-\sum_{i=1}^{p} p_{i} Z_{t-i}-\sum_{i=1}^{q} \phi_{i} a_{t-i}\right.
$$

De tal forma que, para calcular las innovaciones a partir de un conjunto de información de un vector de parámetros desconocidos, se precisan un conjunto de valores iniciales $Z_{0}, Z_{1}, \ldots, Z_{p-1}$ y $a_{0}, a_{1}, \ldots, a_{q-1}$.

En la aplicación, el mecanismo seguido es aproximar las innovaciones imponiendo una serie de condiciones sobre los valores iniciales, con lo que se consigue los denominados estimadores de Mínimos Cuadrados Condicionados y Máximo verosímiles Condicionado. La condición que se aplica generalmente sobre los valores iniciales es que las p primeras observaciones de $\mathrm{Z}_{t}$ son los valores iniciales y las innovaciones previas son cero. Los estimadores Máximo verosímiles condicionados a las $p$ primeras observaciones son iguales a los estimadores Mínimos Cuadrados condicionados.

Existen métodos para alcanzar estimadores Máximos verosímiles no condicionados, basados en la maximización de la función de verosimilitud exacta que se logra como una combinación de la función de verosimilitud condicionada y la función de densidad no condicionada para los valores iniciales.

La parte de validación se procede teniendo en cuenta si las estimaciones de los coeficientes del modelo son significativas y cumplen las condiciones de estacionariedad e invertibilidad a la evaluación de los modelos estimados de los datos que deben satisfacer los parámetros del modelo y si los residuos del modelo tienen un comportamiento similar a las innovaciones, esto es si son ruido blanco.

Antes de todo, se han de realizar los contrastes habituales de significación individual de los coeficientes AR y MA:

$$
\mathrm{B}=\left(\alpha, p_{1}, \ldots, p_{p}, \phi_{1}, \ldots, \phi_{\mathrm{p}}\right)
$$

Para constatar si el modelo planteado está sobre identificado, en otros términos, si se han adjuntado estructura que no es relevante, el caso más habitual de un ARMA (p, q) con constante se plantean los siguientes contrastes:
Ho : $\alpha=0$
frente a
Ha $: \alpha \neq 0$
Ho : $p_{i}=0$
frente a
Ha : $p_{i} \neq 0$
Ho : $\phi \mathrm{i}=0$
frente a 
Comúnmente, la distribución asintótica de los estimadores es la siguiente:

$$
\hat{\beta} i \sim N\left(\beta_{i}, V(\hat{\beta} i)\right) \quad \forall i
$$

Con la varianza dada por la inversa de la matriz de información. De tal forma que, para contrastar la Ho de no significatividad individual de los parámetros se utilizará el estadístico $\mathrm{t}$ habitual que sigue asintóticamente una distribución normal:

$$
\mathrm{t}=\frac{\widehat{\beta} i-0}{\sqrt{V(\widehat{\beta} i)}} \sim \mathrm{N}(0,1)
$$

Se rechazará la hipótesis nula a un nivel de significación $\alpha=5 \%$, cuando:

$$
\left|\frac{\widehat{\beta} i}{\sqrt{V(\widehat{\beta} i)}}\right|>N_{\frac{\alpha}{2}}(0,1) \approx 2
$$

Por otra parte, es importante verificar si las condiciones de estacionariedad e invertibilidad se satisfacen para el modelo propuesto. Para esto, se calculan las raíces del polinomio autorregresivo $\hat{p}(L)=0$, y las raíces del polinomio de medias móviles $\widehat{\phi}(L)=0$. Si alguna de estas raíces está próxima a la unidad podría apuntar a una falta de estacionariedad o invertibilidad.

Finalmente, con el fin de detectar la posible presencia de una correlación excesivamente alta entre las estimaciones de los mismos es oportuno también analizar la matriz de covarianzas entre los parámetros estimados lo que puede ser un indicio de la presencia de factores comunes en el modelo.

Luego de estudiar los coeficientes estimados lo siguiente es analizar los residuos. El análisis de residuos consiste en una serie de contrastes de diagnóstico con el objetivo de determinar si los residuos replican el comportamiento de un ruido blanco, es decir, si su media es cero, su varianza constante y las autocorrelaciones nulas.

Para comprobar si la media es cero se realiza un análisis gráfico, representando los residuos a lo largo del tiempo y observando si los valores oscilan alrededor de cero. De igual manera, se puede llevar a cabo el siguiente contraste de hipótesis: Ho: $\mathrm{E}\left(a_{t}\right)=0$ vs $\mathrm{Ha}=\mathrm{E}\left(a_{t}\right) \neq 0$. El estadístico de contraste se distribuye bajo la hipótesis nula como sigue:

$$
\mathrm{T}=\sqrt{T} \frac{\hat{a}}{\sqrt{\operatorname{Co}(\hat{a})}} \sim N(0,1)
$$

Donde $\hat{a} y \operatorname{Co}(\hat{a})$ son respectivamente la media y la varianza muestrales de los residuos. Mientras que si en el gráfico de los residuos la dispersión de estos es constante deduciremos que la varianza de $a_{t}$ permanece constante.

$\mathrm{Si}$ los residuos se portasen como un ruido blanco, los coeficientes de la FAC y FACP muestrales deben ser prácticamente nulos para todos los retardos. Para corroborarlo se lleva a cabo un contraste de significatividad individual sobre los coeficientes de autocorrelación Ho: $\rho_{k}$ (a) $=0$ versus la alternativa Ha: $\rho_{k}(a) \neq 0$, bajo la hipótesis nula se tiene que:

$$
\widehat{\rho_{k}}(\mathrm{a}) \sim \mathrm{N}\left(0, \frac{1}{T}\right)
$$

Se dirá que $a_{t}$ es un ruido blanco si los coeficientes de autocorrelación estimados están dentro del intervalo de no significación, es decir si:

$$
\left|\widehat{\rho_{k}}(a)\right| \leq \frac{2}{\sqrt{T}}
$$

Para todo k o al menos para el $95 \%$ de los coeficientes estimados.

\subsection{Definición del Modelo Estocástico ARIMA para la morosidad de consumo del Ecuador.}

El modelo estocástico (ARIMA) desarrollado para la morosidad de consumo del Ecuador cubre el periodo 20092019 con frecuencia mensual. El razonamiento del modelo es obtener resultados paralelos a los ofrecidos por el VAR. Se buscan los valores p y q de modo tal que con la menor cantidad de parámetros posible se pueda explicar las series estudiadas sin un elevado sacrificio en términos estadísticos.

\section{RESULTADOS}

La iniciación de la serie al año 2009 tiene aparejada la expectativa deliberada de adentrar el cambio estructural de la caída del precio del petróleo y la apreciación del dólar durante el 2014-2015. El suceso constituye un apremiante caso de estudio, el más característico evento de tensión financiero y económico de la historia reciente y por ello, al mismo tiempo, por su relativa contigüidad es incluido en todos los trabajos concernientes al Ecuador.

Varias de las variables explicativas de la morosidad de consumo resultan significativas a su propia historia, el Pib, la tasa de cambio real además del rezago de la morosidad tiene una magnitud destacable; una variación de $1 \%$ en la Morosidad en $t_{-1}$ genera una variación de la morosidad en t de $0.78 \%$ a su vez una variación del $1 \%$ en la Tasa de Cambio real genera una variación de la morosidad en t de $-1.00 \%$. Los movimientos de la liquidez son sensibles a la inercia de la liquidez rezagada, tipo de cambio real y la morosidad, siendo el impacto más relevante el de la inercia con un coeficiente del $0.99 \%$. Para el PIB las variables significativas son su propio regazo, la liquidez y el precio de petróleo, como era de esperarse una suba en la liquidez y el precio del commodities conlleva un incremento en la actividad productiva, una variación de $1 \%$ en $M_{2}$ genera un incremento del PIB en $0.17 \%$, una variación del precio del petróleo del $1 \%$ deriva, ceteris paribus, en un crecimiento del PIB del $0.10 \%$.

Finalmente, para el Tipo de cambio real son significativas todas las variables a excepción de $M_{2} \mathrm{y}$ morosidad. Una variación del del $1 \%$ de TCR en $t_{-1}$ deriva ceteris paribus, en un crecimiento del TCR del $0.77 \%$, un aumento del $1 \%$ del PIB genera una variación negativa del Tipo de cambio Real del $-0.03 \%$ y un incremento del $1 \%$ del precio del petróleo genera un incremento del TCR del $0.01 \%$. Ver TABLA II 
TABLA II

ESTIMADORES DE LOS PARÁMETROS DEL VAR, SIENDO LOS DESVIIOS ESTÁNDAR LOS VALORES QUE ESTÁN ENTRE PARÉNTESIS Y EL P-VALUE EN CORCHETES

\begin{tabular}{ccccccc}
\hline $\begin{array}{c}\text { Variab } \\
\text { le }\end{array}$ & Moc & $\boldsymbol{M}_{\mathbf{2}}$ & $\boldsymbol{Y}_{\boldsymbol{t}}$ & $\mathbf{T C R}$ & $\mathbf{S t}$ & $\mathbf{R}^{\wedge} \mathbf{2}$ \\
\hline Moc & 0.784136 & - & 0.216321 & - & - & 0.921 \\
& $(0.05958$ & 0.061265 & $(0.08483$ & 1.001088 & 0.018922 & 6 \\
& $0)$ & $(0.03769$ & $6)$ & $(0.40460$ & $(0.02883$ & \\
& {$[0.0000]$} & $6)$ & {$[0.0123]$} & $5)$ & $8)$ & \\
$\boldsymbol{M}_{\mathbf{2}}$ & 0.043712 & 0.996438 & - & 0.225280 & 0.003573 & 0.997 \\
& $(0.01331$ & $(0.00842$ & 0.022425 & $(0.09039$ & $(0.00644$ & 7 \\
& $1)$ & $2)$ & $(0.01895$ & $7)$ & $3)$ & \\
& {$[0.0014]$} & {$[0.0000]$} & $4)$ & {$[0.0143]$} & {$[0.5805]$} & \\
$\boldsymbol{Y}_{\boldsymbol{t}}$ & 0.111771 & 0.172796 & 0.307198 & - & 0.108513 & 0.590 \\
& $(0.06491$ & $(0.04107$ & $(0.09243$ & 0.230655 & $(0.03142$ & 0 \\
& $6)$ & $2)$ & $3)$ & $(0.44083$ & $1)$ & \\
& {$[0.0882]$} & {$[0.0001]$} & {$[0.0012]$} & $8)$ & {$[0.0008]$} & \\
TCR & - & 0.004461 & - & 0.774992 & 0.010831 & 0.945 \\
& 0.016416 & $(0.00550$ & 0.030113 & $(0.05905$ & $(0.00420$ & 2 \\
& $(0.00869$ & $2)$ & $(0.01238$ & $0)$ & $9)$ & \\
& $5)$ & {$[0.4194]$} & $1)$ & {$[0.0000]$} & {$[0.0115]$} & \\
& {$[0.0619]$} & \multicolumn{0}{c}{$[0.0168]$} \\
\hline
\end{tabular}

Fuente: Elaboración propia en base a datos del Banco Central del Ecuador.

Dado que los criterios de indagación utilizados (FRE y AIC) reflejan que dos es el numero recomendable de rezagos, se reestimó el modelo con los lags que sugieren los criterios. Por otro lado, el criterio (LR) aconseja la utilización de siete rezagos y el criterio (SC) un rezago presunción validada por ser uno de los criterios que suelen elegir especificaciones con una pequeña consideración de rezagos. Pasan a tener una magnitud relevante los coeficientes asociados del cotejo del modelo con uno a dos rezagos. Ver TABLA III.

TABLA III

VAR CRITERIO DE SELECCIÓN DE ORDEN DE RETRASO

\begin{tabular}{lllll}
\hline \multicolumn{1}{c}{ Lag } & \multicolumn{1}{c}{ LR } & \multicolumn{1}{c}{ FRE } & \multicolumn{1}{c}{ AIC } & \multicolumn{1}{c}{ SC } \\
\hline $\mathbf{0}$ & $\mathrm{Na}$ & 314.4215 & 17.1022 & $17.3159^{*}$ \\
$\mathbf{1}$ & 49.33064 & 253.8080 & 16.8874 & 17.1465 \\
$\mathbf{2}$ & 44.54284 & $211.2812^{*}$ & $16.7066^{*}$ & 17.7701 \\
$\mathbf{3}$ & 18.29099 & 237.1769 & 16.8119 & 18.3078 \\
$\mathbf{4}$ & 24.58513 & 234.8434 & 16.8300 & 18.7533 \\
$\mathbf{5}$ & 19.20655 & 266.5330 & 16.9038 & 19.2545 \\
$\mathbf{6}$ & $29.39742^{*}$ & 249.9247 & 16.8172 & 19.5952 \\
$\mathbf{7}$ & 25.54160 & 244.3426 & 16.7635 & 19.9690 \\
$\mathbf{8}$ & 15.86710 & 275.2671 & 16.8409 & 20.4738 \\
$\mathbf{9}$ & 20.54349 & 284.7270 & 16.8201 & 20.8803 \\
$\mathbf{1 0}$ & 18.35433 & 303.4228 & 16.8135 & 21.3011 \\
\hline
\end{tabular}

Fuente: Elaboración propia en base a datos del Banco Central del Ecuador

\subsection{Estimación del var.}

Para el VAR completo, además para las cuatro ecuaciones tomadas individualmente se rechaza la hipótesis nula ajustado al test de restricciones de exclusión. El total conjunto de los parámetros poblaciones con un $100 \%$ de confianza tiene valor nulo. Ver TABLA IV.

TABLA IV

VAR PRUEBAS DE WALD DE EXCLUSIÓN DE RETRASO

\begin{tabular}{llll}
\hline Ecuación & Lag & Ch2(mo) & Prob>ch2 \\
\hline $\mathbf{1}$ & 1 & $(448.2273)$ & {$[0.0000]$} \\
$\mathbf{2}$ & 1 & $(29048.62)$ & {$[0.0000]$} \\
$\mathbf{3}$ & 1 & $(135.2971)$ & {$[0.0000]$} \\
$\mathbf{4}$ & 1 & $(498.4742)$ & {$[0.0000]$} \\
Todas & 1 & $(61068.72)$ & {$[0.0000]$} \\
\hline
\end{tabular}

Fuente: Elaboración propia en base a datos del Banco Central del Ecuado
La observación residual es un material agregado que permite establecer si el modelo estuvo cabalmente desarrollado el cual muestra el contraste por correlación serial del estadístico LM de los residuos del VAR (2) cuya hipótesis nula: No hay correlación serial en el lag j. No existe axioma para refutar la hipótesis de ausencia de correlación en este proceso. Ver TABLA V.

TABLA V

VAR PRUEBAS DE LM DE CORRELACIÓN SERIAL RESIDUAL

\begin{tabular}{lllll}
\hline Lag & LRE*stat & Prob & Rao F-stat & Prob \\
\hline $\mathbf{1}$ & 16.2592 & 0.4350 & 1.02130 & 0.4355 \\
$\mathbf{2}$ & 28.0446 & 0.0312 & 1.81203 & 0.0314 \\
$\mathbf{3}$ & 19.9501 & 0.2225 & 1.26424 & 0.2229 \\
$\mathbf{4}$ & 25.7217 & 0.0581 & 1.65269 & 0.0583 \\
$\mathbf{5}$ & 10.1021 & 0.8612 & 0.62532 & 0.8614 \\
$\mathbf{6}$ & 24.2071 & 0.0851 & 1.54973 & 0.0854 \\
$\mathbf{7}$ & 20.5569 & 0.1962 & 1.30458 & 0.1966 \\
$\mathbf{8}$ & 15.2863 & 0.5038 & 0.95796 & 0.5043 \\
$\mathbf{9}$ & 16.7755 & 0.4003 & 1.05503 & 0.4008 \\
$\mathbf{1 0}$ & 23.0889 & 0.1114 & 1.47418 & 0.1117 \\
\hline
\end{tabular}

Fuente: Elaboración propia en base a datos del Banco Central del Ecuador

El proceso es dinámicamente sólido y estable garantizando la estacionariedad de las variables al ser todos los autovalores mostrados en valor absoluto menor a la unidad, es más, si no fuese estacionaria una o alguna de las variables endógenas quiere decir que una perturbación transitoria en alguna de las mismas implicaría una alteración sobre los valores futuros del proceso y dicha dinámica no revertiría a la media. Así además se muestra que los errores en la estimación del VAR están normalmente distribuidos y son homocedásticos mediante las pruebas de normalidad y heterocedasticidad. Ver TABLA VI.

TABLA VI RAÍCES DEL POLINOMIO CARACTERÍSTICO

\begin{tabular}{ll}
\hline Autovalores & Módulo \\
\hline $\mathbf{0 . 9 8 9 8 8 8}$ & 0.989888 \\
$\mathbf{0 . 9 5 3 9 7 4}$ & 0.953974 \\
$\mathbf{0 . 6 5 2 6 1 6}$ & 0.652616 \\
$\mathbf{0 . 2 6 6 2 8 5}$ & 0.266285 \\
\hline
\end{tabular}

Fuente: Elaboración propia en base a datos del Banco Central del Ecuador

\subsection{Funciones Impulso Respuesta.}

Las funciones de impulso respuesta ortogonalizadas permiten determinar cómo se propaga el shock exógeno que no tiene que ver con las variables exógenas, sino con un shock exógeno sobre las variables endógenas a través del sistema. En $\mathrm{t}=0$ se supone un impulso de las cuatro variables equivalente en magnitud a las distintas columnas de la matriz de varianzas y covarianzas de los errores que surge de la descomposición de Cholesky.

Las funciones de impulso respuesta permiten ver la prontitud del ajuste. Resultados expuestos en la Fig 1, la línea central es la función de respuesta al impulso, mientras que las líneas de los extremos de color rojo son simplemente los intervalos de confianza del $95 \%$, por lo que su función de respuesta al impulso siempre estuvo dentro del intervalo de confianza del 95\%.

La conducta del indicador de calidad de cartera frente a un choque del incremente del PIB es uno de los resultados más interesantes del presente estudio dado que da como consecuencia a un acentuado detrimento hasta el séptimo 
periodo resultado que puede considerarse contraintuitivo ya que la expectativa de la actividad económica es que mejore la capacidad de pago de los individuos y el indicador de morosidad se reduzca. A pesar de ello, esto puede explicarse por la saturación que se puede desplegar en estos créditos y por perjuicio de los rendimientos en periodos anteriores.

Fig 1: Shock de $M_{2}$, Pib, Tcr y como morosidad de consumo responde:
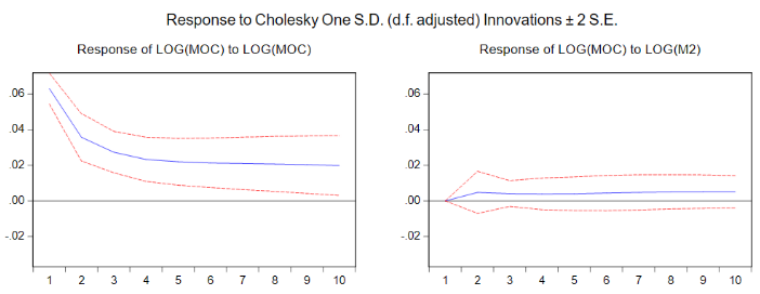

Response of LOG(MOC) to LOG(MT) Response of LOG(MOC) to LOG(TCR)
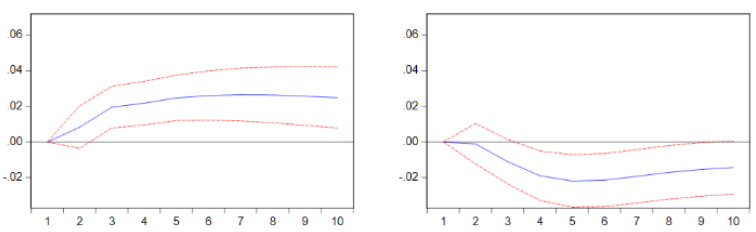

Fuente: Elaboración propia en base a datos del Banco Central del Ecuador

Se contemplaron igualmente los resultados para este estudio al agregar la $M_{2}$ como medida de liquidez del sistema financiero. Entre los resultados encontrados para Ecuador en las distintas especificaciones del periodo estimado inverso a lo encontrado en la literatura es que la liquidez no soporta la influencia en la calidad de cartera de impago de consumo.

Inicialmente un shock de desviación estándar (innovación) del tipo de cambio real multilateral no tiene una marca considerable en la morosidad de consumo en el periodo uno, no obstante, a partir del segundo periodo provoca una contestación positiva de la calidad de cartera en donde permanece en la zona negativa hasta alrededor del octavo periodo y con tendencias crecientes. Esto puede explicarse al mejorar las condiciones del mercado financiero.

\subsection{Descomposición de la varianza}

Inspeccionar la contribución marginal de los shocks asociados a cada una de las variables es permitido mediante la descomposición de la varianza sobre la variabilidad integral de las restantes variables endógenas en una extensión predeterminada el cual dentro del presente estudio es de diez periodos. La exploración de esta técnica permite diferenciar la epístola de movimientos sobre las variables endógenas que corresponden a su propio shock de los incrementos de otras variables del VAR.

La morosidad de consumo responde en el periodo 10 un $54.55 \%$ ante un impulso de sí mismo, un $1.13 \%$ ante un impulso de la liquidez del sistema Financiero, un 29.23\% ante un impulso del Producto Interno Bruto, un 15.08\% ante un impulso del tipo de cambio real multilateral. La liquidez del sistema financiero responde en el periodo 10 a un $67.03 \%$ ante un impulso de sí misma, un $19,91 \%$ ante un impulso de la morosidad, un $0.20 \%$ ante el PIB y un $12.84 \%$ ante la Tasa de Cambio Real. El PIB responde un $79.81 \%$ ante sí mismo, un $13.85 \%$ frente a la morosidad, un 5.43 frente a $M_{2}$ y un $0.89 \%$ frente al tipo de cambio real. Finalmente, el tipo de cambio real multilateral responde un $63,73 \%$ frente a sí mismo, un $24.39 \%$ frente al PIB, un $1.33 \%$ frente a $M_{2}$ y un $10.53 \%$ frente a la morosidad (en el anexo se adjuntan las tablas de resultados).

\subsection{Causalidad de Granger}

La historia de las variables causales afecta el valor actual de la variable respuesta una vez controlada la relación causa- efecto por la memoria agregada de esta última. Es lo que el test de causalidad busca instituir. Para la ecuación de la morosidad de consumo la historia del tipo de cambio real, el Producto Interno Bruto y la Liquidez Total causan en el sentido de Granger a la tasa de variación presente de la morosidad de consumo, lo mismo ocurre con la historia conjunta del total de las variables.

De igual forma, para la segunda ecuación, la historia de la tasa de variación de la morosidad, el tipo de cambio real y de la totalidad de las variables tomadas en conjunto, causan en el sentido de Granger a la tasa de variación de la Liquidez total del sistema financiero. Para la tercera ecuación, la historia de la tasa de variación de $M_{2}$ y de la totalidad de las variables tomadas en conjunto causan en el sentido Granger a la tasa de variación presente del PIB. Para la cuarta ecuación, la historia de la tasa de variación de la morosidad, el Producto Interno Bruto y de la totalidad de las variables tomadas en conjunto causan en el sentido de Granger a la tasa de variación presente del tipo de cambio real. Ver TABLA VII.

TABLA VII

PRUEBA DE WALD DE CAUSALIDAD Y EXOGENEIDAD DE GRANGER

\begin{tabular}{|c|c|c|c|}
\hline Ecuación & Excluyendo & Chi-sq & Prob \\
\hline \multirow{4}{*}{$\begin{array}{l}\text { Morosidad } \\
\text { Segmento } \\
\text { Consumo } \\
\text { (MOC) }\end{array}$} & $M_{2}$ & 5.457907 & 0.0653 \\
\hline & YT & 10.44484 & 0.0054 \\
\hline & TCR & 8.166483 & 0.0169 \\
\hline & Todas & 24.46311 & 0.0004 \\
\hline \multicolumn{4}{|l|}{ Ecuación } \\
\hline \multirow[t]{4}{*}{$M_{2}$} & $\begin{array}{l}\text { Morosidad } \\
\text { consumo }\end{array}$ & 9.850011 & 0.0073 \\
\hline & YT & 0.895536 & 0.6391 \\
\hline & TCR & 8.075217 & 0.0176 \\
\hline & Todas & 13.39547 & 0.0372 \\
\hline \multicolumn{4}{|l|}{ Ecuación } \\
\hline \multirow[t]{4}{*}{ YT } & $\begin{array}{l}\text { Morosidad } \\
\text { consumo }\end{array}$ & 4.697872 & 0.0955 \\
\hline & $M_{2}$ & 10.62333 & 0.0049 \\
\hline & TCR & 1.047632 & 0.5923 \\
\hline & Todas & 20.53375 & 0.0022 \\
\hline \multicolumn{4}{|l|}{ Ecuación } \\
\hline \multirow[t]{4}{*}{ TCR } & $\begin{array}{l}\text { Morosidad } \\
\text { consumo }\end{array}$ & 9.808470 & 0.0074 \\
\hline & $M_{2}$ & 0.310526 & 0.8562 \\
\hline & YT & 8.434134 & 0.0147 \\
\hline & Todas & 21.87743 & 0.0013 \\
\hline
\end{tabular}

Fuente: Elaboración propia en base a datos del Banco Central del Ecuador. 


\subsection{Identificación del Modelo ARIMA para la morosidad de consumo del Ecuador}

En lo que resta del análisis, entonces, se va a utilizar la serie a la cual se le aplica logaritmos y las diferencia. Lo que se está buscando es que las funciones muestrales reflejen con cierta precisión los patrones teóricos para estar, de alguna manera, seguros de que se está yendo en la dirección correcta en la construcción de los modelos ARIMA. Ver Fig 2.

Fig 2: Correlograma de la serie diferenciada de los logaritmos de la Morosidad de Consumo:

\begin{tabular}{|c|c|c|c|c|c|}
\hline Autocorrelation & Partial Correlation & $A C$ & PAC & Q-Stat & Prob \\
\hline 口 1 & 口 1 & $1-0.229$ & -0.229 & 5.7010 & 0.017 \\
\hline 口। & & $2-0.262$ & -0.332 & 13.276 & 0.001 \\
\hline $1 \square$ & וb 1 & $\begin{array}{ll}3 & 0.267\end{array}$ & 0.132 & 21.227 & 0.000 \\
\hline$1 \longdiv { 1 1 }$ & 1 & $4 \quad 0.052$ & 0.091 & 21.531 & 0.000 \\
\hline It 1 & $1 \longdiv { 1 }$ & $5-0.139$ & 0.009 & 23.729 & 0.000 \\
\hline $1 \square$ & $1 \boxminus$ & $\begin{array}{ll}6 & 0.263\end{array}$ & 0.272 & 31.652 & 0.000 \\
\hline 吅 & $1 \longdiv { 1 }$ & $7-0.145$ & -0.104 & 34.074 & 0.000 \\
\hline ו ו & 1 & $8 \quad 0.113$ & 0248 & 35.559 & 0.000 \\
\hline
\end{tabular}

Fuente: Elaboración propia en base a datos del Banco Central del Ecuador.

Para la serie de la morosidad del segmento consumo el pico de la función de correlación más importante se encuentra en el tercer y sexto rezago por lo cual se podría incluir en los modelo de elección un componente AR(3) o AR (6), mientras que la función de autocorrelación parcial presenta picos relevantes en el sexto y octavo rezago, por lo cual se considera en el modelo un componente $\mathrm{MA}(6)$ o MA(8).

En la siguiente TABLA VIII se encuentra los resultados de la estimación de la morosidad de Consumo del Ecuador.

TABLA VIII

PRESULTADOS DE LA TRANSFORMACIÓN ESTACIONARIA Zt2 $=\Delta \ln (\mathrm{Moc})$ DE LA SERIE MOROSIDAD DE CONSUMO

\begin{tabular}{lllll}
\hline $\mathbf{Z t}=$ & $\operatorname{ARIMA}(3$, & $\operatorname{ARIMA}(1$, & $\operatorname{ARIMA}(6$, & $\operatorname{ARIMA(6,}$ \\
$\Delta \ln ($ Moc $)$ & $\mathbf{1 , 0 )}$ & $\mathbf{1 , 6})$ & $\mathbf{1 , 6})$ & $\mathbf{1 , 8})$ \\
\hline Coeficien & 1 & 2 & 2 & 2
\end{tabular}

tes

significat

ivos

$\begin{array}{lllll}\text { Sigma }^{2} & 0.00455 & 0.004558 & 0.00276 & 0.00418\end{array}$

(Volatilid

ad)

$\begin{array}{lllll}\text { Sum } & 0.48317 & 0.483143 & 0.29275 & 0.4433\end{array}$

squred

resid

$\begin{array}{lllll}\text { Adj. } \mathbf{R}^{2} & 0.06563 & 0.05662 & 0.42838 & 0.1342\end{array}$

$\begin{array}{lllll}\text { AIC } & -2.5130 & -2.4954 & -2.84310 & -2.5662\end{array}$

$\begin{array}{lllll}\text { SBIC } & -2.4627 & -2.4200 & -2.7677 & -2.4908\end{array}$

Fuente: Elaboración propia en base a datos del Banco Central del Ecuador.

El modelo ARIMA $(6,1,6)$ permite percibir buena parte del comportamiento de la serie. En la elección del modelo se consideró aquel que tenga la mayor cantidad de coeficientes significativos, la menor volatilidad, el mayor R cuadrado ajustado y los criterios de información Akaike (AIC) y Schwarz (SBIC) más bajos. En definitiva, el modelo queda expresado como:

$$
\text { DLMOC }=(0.9467) \text { DLMOC }_{t-6}+(-0.8934) \mathrm{e}_{t-6}+\mathrm{e}_{t}
$$

Donde en todos los casos $\mathrm{e}_{t}$ son los residuos estimados. Es relevante llevar a cabo un diagnostico general del modelo obtenido. Para ello, en primer lugar, se evaluó la significatividad individual de los residuos utilizando sus correlogramas. Como se puede ver en la Fig 3 los residuos caen dentro de las bandas, por lo cual se puede asumir que estos valores son individualmente significativos. La impresión general, entonces es que los residuos generados por las estimaciones ARIMA son puramente aleatorios. En la Fig 4 del comportamiento de los residuos puede verse como de hecho parecen comportarse como ruido blanco.

Fig 3: Contraste de diagnóstico: Correlograma de residuos del modelo ARIMA Moc:

\begin{tabular}{|c|c|c|c|c|c|c|}
\hline Autocorrelation & Partial Correlation & & $\mathrm{AC}$ & PAC & Q-Stat & Prob \\
\hline וך ו & וp ו & 1 & 0.109 & 0.109 & 1.2947 & 0.255 \\
\hline $1 \sqrt{1}$ & $1 \sqrt{1}$ & 2 & 0.051 & 0.040 & 1.5863 & 0.452 \\
\hline I 1 & 吅 1 & 3 & -0.136 & -0.147 & 3.6347 & 0.304 \\
\hline 171 & 17 & 4 & -0.013 & 0.016 & 3.6524 & 0.455 \\
\hline 101 & 101 & 5 & -0.082 & -0.069 & 4.4075 & 0.492 \\
\hline 171 & 1 1 & 6 & 0.069 & 0.068 & 4.9492 & 0.550 \\
\hline $10 \sqrt{1}$ & $10 \sqrt{1}$ & 7 & -0.072 & -0.083 & 5.5503 & 0.593 \\
\hline 1 1 & 1 1 1 & 8 & 0.053 & 0.046 & 5.8783 & 0.661 \\
\hline
\end{tabular}

Fuente: Elaboración propia en base a datos del Banco Central del Ecuador

Fig 4: Comportamiento de los residuos del modelo ARIMA Moc:

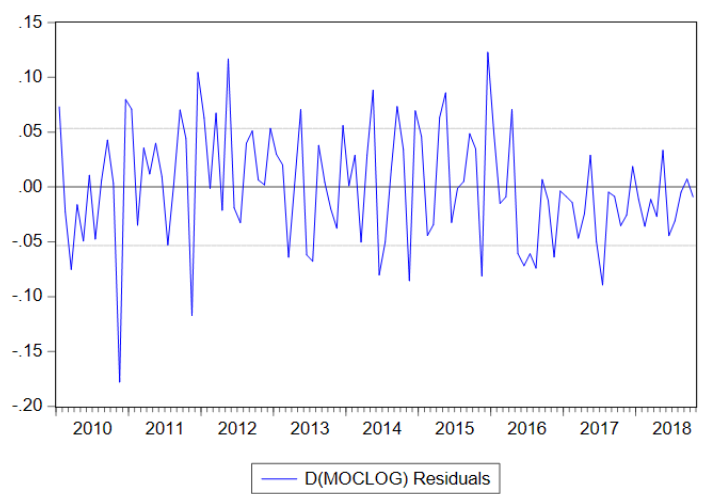

Fuente: Elaboración propia en base a datos del Banco Central del Ecuador

\section{CONCLUSIONES}

La técnica econométrica de vectores autorregresivos (VAR) fue elegida en el informe del actual artículo el cual se centralizó en el tratamiento del modelo macroeconómico. Esta designación supera a otras metodologías sugeridas en la literatura que oportunamente fueron indagadas en el marco teórico existiendo como corolario una evidencia juiciosa para puntualizar que la conjetura de investigación se cumple.

Mediante la elección del conjunto de variables apropiadas surge el esmero de ejecutar los objetivos a través de los modelos VAR ya que por su estructura son más versátiles para el investigador. En este artículo durante el montaje del diseño y aplicación del VAR se presentaron las siguientes dificultades, una de ellas es el escogimiento de variables que se vio obstaculizada por la información disponible. A ello, se añade la estructura de los cambios de métodos que van surgiendo en la medición de las variables 
que dificulta aún más la representación de las series de larga continuidad. Sin la cuantía ideal de observaciones surge la problemática de explorar empíricamente el impacto de ciertas teorías económicas.

Un modelo con cuatro variables endógenas ha conseguido proveer este artículo, de las cuales las variables son la morosidad de consumo (Moc), la Liquidez Total (M2), el Producto Interno Bruto (PIB), el Tipo de cambio Real (TCR), incluso se realizó la nominación de la variable exógena del VAR optándose por el Precio del Petróleo ecuatoriano cuyo movimiento y evolución dentro del periodo reverenciado respondió como hacedor de política económica y cuya perturbación surge de los mercados internacionales, al mismo tiempo, de impactar en la liquidez del sector público como ocurrió con el desplome de precios durante el 2014 y 2015 provocado porque la demanda mundial de este commodity disminuyó.

Resultan interesantes los hallazgos de este artículo en cuanto se evidencia tres hechos estilizados para Ecuador sobre los determinantes de la calidad de cartera de morosidad de consumo del Ecuador, de los resultados obtenidos muestran que el comportamiento del indicador de calidad de cartera frente a PIB deriva en una marcada disminución y deterioro de cartera, este efecto puede considerarse contraintuitivo que se espera que la actividad económica mejore el volumen de pago de los individuos y se reduzcan los indicadores de morosidad. Sin embargo, la saturación que se puede ostentar en estos créditos sumado el detrimento de los rendimientos en periodos anteriores puede explicar este efecto, mientras tanto después del séptimo periodo la calidad de cartera prospera. Paradójicamente contrario a lo encontrado en la literatura, la liquidez total (M2) no soporta la atribución en la calidad de cartera. Por otra parte, inicialmente no tiene una marcada transcendencia un shock de desviación estándar del tipo de cambio real multilateral en la morosidad de consumo, sin embargo, provoca una respuesta positiva a la calidad de cartera de consumo a partir del segundo periodo.

Los resultados de este estudio son, en general, bastantes ilustrativos. Sería interesante en futuros trabajos permitirse examinar el funcionamiento de estos modelos con otras variables económicas y otras frecuencias de datos. Por otro lado, inevitablemente los resultados estarán sesgados por el juicio competente del profesional, las decisiones impactarán en la designación de las variables a modelar, el tipo de modelo, la elección del ciclo y periodicidad a estimar entre otras cosas. A pesar de ello, la heterogeneidad en la respuesta a shocks en componentes macroeconómicos pauta los grandes retos que implicará la coordinación de la política económica.

\section{REFERENCIAS}

[1] L. E. Peñafiel, "La Dinámica Macroeconómica y la morosidad del sistema financiero del Ecuador (2009-2018)," Cuest. Económica, vol. 29 , no. $1,2019$.

[2] [2] FMI, "Nota Técnica: Estabilidad del Sector Financiero aplicado a Argentina," vol. 35, no. 1, pp. 1-21, 2012.

[3] [3] Randall Kroszner, "Non-Performing Loans, Monetary Policy and Deflation: The Industrial Country Experience," 2002.

[4] [4] D. Rosch, "Correlations and business cycles of credit risk: Evidence from bankruptcies in Germany," Financ. Mark. Portf. Manag., vol. 17, no. 3, pp. 309-331, 2003.

[5] [5] J. Marcucci and M. Quagliariello, "CREDIT RISK AND BUSINESS CYCLE OVER DIFFERENT REGIMES Juri,” 2006.
[6] [6] M. Vallcorba and J. Delgado, "Determinantes de la Morosidad bancaria en una Economía Dolarizada: El caso Uruguayo," Banco de España, vol. 24, pp. 8-14, 2007.

[7] [7] R. Kräussl, A. B. Monteiro, S. J. Koopman, and A. Lucas, "Credit cycles and macro fundamentals," J. Empir. Financ., vol. 16, no. 1, pp. 42-54, 2009.

[8] [8] R. Kattai, Credit Risk Model for the Estonian Banking Sector. 2010.

[9] [9] J. Crook and T. Bellotti, "Time varying and dynamic models for default risk in consumer loans," J. R. Stat. Soc. Ser. A Stat. Soc., vol. 173, no. 2, pp. 283-305, 2010.

[10] [10]R. G. Martínez, "Un Modelo de Alerta Temprana basado en Análisis Factorial Robusto y Funciones Discriminantes Bayesianas - Una Aplicación al Sistema Financiero de Bolivia," 2011.

[11] [11]M. B. E. das Neves and J. T. M. Marins, "Credit Default and Business Cycles: an investigation of this relationship in the brazilian corporate credit market," Banco Cent. do Bras., pp. 132,2013

[12] [12]A. A. Fajardo Moreno, "Impacto del comportamiento macroeconómico sobre la morosidad de la cartera de consumo en Colombia," 2016.

[13] [13]M. Alexandre, G. Silva, and T. Cotrim, "Default contagion among credit modalities: evidence from Brazilian data," 76859, 2017.

[14] [14]M. Betti, "Pruebas de estrés en entidades financieras. El modelo de vectores autorregresivos como metodología para la generación de escenarios macreocnomómicos,” Buenos Aires, pp. $1-20,2018$

[15] [15]L. Schou-Zibell, J. R. Albert, and S. Lei Lei, "A Macroprudential Framework for Monitoring and Examining Financial Soundness," 2010.

[16] [16]M. Cicowiez and L. Di Gresia, "Equilibrio general computado: Descripción de la metodología," Ser. Trab. Docentes, no. 7,2004

[17] [17]E. Cabezas, "Un modelo de equilibrio general dinámico y estocástico (DSGE) para la economía ecuatoriana," Cuest. Económicas, vol. $26 \mathrm{~N}^{\circ} 1$, pp. 11-58, 2016.

[18] [18]A. Martínez, "Modelos Econométricos para determinar el comportamiento de la cartera comercial de los bancos privados grandes ecuatorianos en el periodo 2007-2015,” 2018.

[19] [19]Oesterreichische Nationalbank (ONB), Risk Assessment and Stress Testing for the Austrian Banking System: Model Documentation. Vienna, 2006.

[20] [20]J. Wooldridge, "Methods in applied econometrics," Am. Econ. Rev., vol. 93, no. 2, 2012.

[21] [21]D. Gujarati and D. Porter, Econometría, Quinta Edi. Mexico: Mc Graw Hill, 2013.

[22] [22]M. Malik and L. Thomas, "Modelling Credit Risk in portfolios of consumer loans: Transition Matrix Model for Consumer Credit Ratings," pp. 1-21, 2010.

[23] [23]C. A. Sims, "Macroeconomics and Reality," Econometrica, vol. 48 , no. 1 , p. 1,1980

[24] [24]W. Ender, "APPLIED ECONOMETRIC TIME SERIES," $J$. Hered., vol. 90, no. 1, pp. 263-264, 2015.

[25] [25]P. González, Análisis de series temporales económicas: modelos ARIMA. 2007.

[26] [26]J. Hernandez, "Modelación ARIMA," Economiamexicana, pp. 41-78, 1994. 


\section{ANEXOS}

DESCOMPOSICIÓN DE LA VARIANZA PARA LA MOROSIDAD DE CONSUMO

\begin{tabular}{llllll}
\hline $\begin{array}{l}\text { Period } \\
\mathbf{0}\end{array}$ & $\mathbf{S . E}$ & $\begin{array}{l}\log (\mathbf{M o} \\
\mathbf{c})\end{array}$ & $\log \left(M_{2}\right)$ & $\log (\mathbf{Y t})$ & $\begin{array}{l}\log (\mathbf{T c} \\
\mathbf{r})\end{array}$ \\
\hline $\mathbf{1}$ & 0.06301 & 100.0000 & 0.000000 & 0.000000 & 0.00000 \\
& 8 & & 0 & 0 & 0 \\
$\mathbf{2}$ & 0.07308 & 98.27010 & 0.424116 & 1.286992 & 0.01879 \\
& 3 & & & & 3 \\
$\mathbf{3}$ & 0.08133 & 90.69054 & 0.595906 & 6.830886 & 1.88276 \\
& 1 & & & 5 \\
$\mathbf{4}$ & 0.08950 & 81.66932 & 0.684424 & 11.58644 & 6.05981 \\
& 6 & & & & 4 \\
$\mathbf{5}$ & 0.09798 & 73.15660 & 0.735020 & 16.04533 & 10.0630 \\
& 9 & & & & 5 \\
$\mathbf{6}$ & 0.10589 & 66.69821 & 0.800692 & 19.78987 & 12.7021 \\
& 3 & & & & 2 \\
$\mathbf{7}$ & 0.11296 & 62.08037 & 0.882087 & 22.95006 & 14.0874 \\
& 0 & & & & 9 \\
$\mathbf{8}$ & 0.11917 & 58.79726 & 0.970717 & 25.51833 & 14.7136 \\
& 4 & & & & 9 \\
$\mathbf{9}$ & 0.12466 & 56.39129 & 1.055678 & 27.58266 & 14.9703 \\
& 3 & & & & 6 \\
$\mathbf{1 0}$ & 0.12956 & 54.55112 & 1.130967 & 29.23518 & 15.0827 \\
& 4 & & & & 4 \\
\hline
\end{tabular}

Fuente: Elaboración propia en base a datos del Banco Central del Ecuador

DESCOMPOSICIÓN DE LA VARIANZA PARA $M_{2}$

\begin{tabular}{cccccc}
\hline $\begin{array}{c}\text { Period } \\
\mathbf{0}\end{array}$ & $\mathbf{S . E}$ & $\begin{array}{c}\log (\mathbf{M o} \\
\mathbf{c})\end{array}$ & $\begin{array}{c}\log \left(M_{2}\right. \\
)\end{array}$ & $\mathbf{L o g}(\mathbf{Y t})$ & $\mathbf{L o g}(\mathbf{T c r})$ \\
\hline $\mathbf{1}$ & 0.06301 & 50.62881 & 49.3711 & 0.00000 & 0.000000 \\
& 8 & & 9 & 0 & 0 \\
$\mathbf{2}$ & 0.07308 & 42.06347 & 57.1453 & 0.38386 & 0.407312 \\
& 3 & & 5 & 5 & \\
$\mathbf{3}$ & 0.08133 & 35.90522 & 60.6741 & 0.28605 & 3.134560 \\
& 1 & & 7 & 1 & \\
$\mathbf{4}$ & 0.08950 & 31.05190 & 62.2728 & 0.22536 & 6.449858 \\
& 6 & & 8 & 6 & \\
$\mathbf{5}$ & 0.09798 & 27.57775 & 63.0272 & 0.18730 & 9.207682 \\
& 9 & & 7 & 0 & \\
$\mathbf{6}$ & 0.10589 & 25.12423 & 63.6919 & 0.16127 & 11.02253 \\
& 3 & & 6 & 8 & \\
$\mathbf{7}$ & 0.11296 & 23.34924 & 64.4493 & 0.14638 & 12.05504 \\
& 0 & & 3 & 6 & \\
$\mathbf{8}$ & 0.11917 & 21.98939 & 65.2935 & 0.14498 & 12.57209 \\
& 4 & & 3 & 7 & \\
$\mathbf{9}$ & 0.12466 & 20.87754 & 66.1697 & 0.16298 & 12.78969 \\
& 3 & & 9 & 2 & \\
$\mathbf{1 0}$ & 0.12956 & 19.91693 & 67.0323 & 0.20560 & 12.84509 \\
& 4 & & 7 & 9 & \\
\hline
\end{tabular}

Fuente: Elaboración propia en base a datos del Banco Central del Ecuador

DESCOMPOSICIÓN DE LA VARIANZA PARA EL PIB

\begin{tabular}{cccccc}
\hline $\begin{array}{c}\text { Period } \\
\mathbf{0}\end{array}$ & $\mathbf{S . E}$ & $\begin{array}{c}\log (\mathbf{M o c} \\
\mathbf{H}\end{array}$ & $\begin{array}{c}\log \left(M_{2}\right. \\
)\end{array}$ & $\mathbf{L o g}(\mathbf{Y t})$ & $\begin{array}{c}\log (\mathbf{T c r} \\
\mathbf{~}\end{array}$ \\
\hline $\mathbf{1}$ & 0.06301 & 14.24310 & 3.72441 & 82.0324 & 0.00000 \\
& 8 & & 9 & 8 & 0 \\
$\mathbf{2}$ & 0.07308 & 13.60455 & 4.12758 & 81.7212 & 0.54658 \\
& 3 & & 3 & 8 & 6 \\
$\mathbf{3}$ & 0.08133 & 13.03568 & 4.38566 & 82.0554 & 0.52317 \\
& 1 & & 4 & 8 & 7 \\
$\mathbf{4}$ & 0.08950 & 13.07873 & 4.65950 & 81.7293 & 0.53342 \\
& 6 & & 6 & 4 & 3 \\
$\mathbf{5}$ & 0.09798 & 13.22038 & 4.85673 & 81.3786 & 0.54424 \\
& 9 & & 9 & 3 & 6 \\
$\mathbf{6}$ & 0.10589 & 13.38605 & 5.01796 & 81.0580 & 0.53790 \\
& 3 & & 5 & 8 & 0 \\
$\mathbf{7}$ & 0.11296 & 13.53463 & 5.14403 & 80.7568 & 0.56444 \\
& 0 & & 6 & 9 & 4 \\
$\mathbf{8}$ & 0.11917 & 13.65779 & 5.24975 & 80.4450 & 0.54738 \\
& 4 & & 0 & 7 & 8 \\
$\mathbf{9}$ & 0.12466 & 13.76147 & 5.34388 & 80.1276 & 0.76700 \\
& 3 & & 6 & 4 & 8 \\
$\mathbf{1 0}$ & 0.12956 & 13.85359 & 5.43339 & 79.8195 & 0.89346 \\
& 4 & & 5 & 5 & 0 \\
\hline
\end{tabular}

Fuente: Elaboración propia en base a datos del Banco Central del Ecuador
DESCOMPOSICIÓN DE LA VARIANZA PARA EL TIPO DE CAMBIO REAL

\begin{tabular}{|c|c|c|c|c|c|}
\hline $\begin{array}{c}\text { Period } \\
\text { o } \\
\end{array}$ & S.E & $\begin{array}{c}\log (\text { Moc } \\
)\end{array}$ & $\begin{array}{c}\log \left(M_{2}\right. \\
)\end{array}$ & $\log (Y t)$ & $\begin{array}{c}\text { Log(Ter } \\
)\end{array}$ \\
\hline 1 & $\begin{array}{c}0.06301 \\
8\end{array}$ & 0.134563 & $\begin{array}{c}0.75118 \\
0\end{array}$ & $\begin{array}{c}0.20957 \\
4\end{array}$ & $\begin{array}{c}98.9046 \\
8\end{array}$ \\
\hline 2 & $\begin{array}{c}0.07308 \\
3\end{array}$ & 0.626077 & $\begin{array}{c}0.50170 \\
7\end{array}$ & $\begin{array}{c}3.34268 \\
6\end{array}$ & $\begin{array}{c}95.5295 \\
3\end{array}$ \\
\hline 3 & $\begin{array}{c}0.08133 \\
1\end{array}$ & 1.661514 & $\begin{array}{c}0.36069 \\
8\end{array}$ & $\begin{array}{c}7.59881 \\
5\end{array}$ & $\begin{array}{c}90.3789 \\
7\end{array}$ \\
\hline 4 & $\begin{array}{c}0.08950 \\
6\end{array}$ & 3.136507 & $\begin{array}{c}0.42841 \\
0\end{array}$ & $\begin{array}{c}12.3175 \\
9\end{array}$ & $\begin{array}{c}84.1174 \\
9\end{array}$ \\
\hline 5 & $\begin{array}{c}0.09798 \\
9\end{array}$ & 4.756960 & $\begin{array}{c}0.62780 \\
2\end{array}$ & $\begin{array}{c}16.2220 \\
8\end{array}$ & $\begin{array}{c}78.3931 \\
6\end{array}$ \\
\hline 6 & $\begin{array}{c}0.10589 \\
3\end{array}$ & 6.295666 & $\begin{array}{c}0.84822 \\
7\end{array}$ & $\begin{array}{c}19.0305 \\
0\end{array}$ & $\begin{array}{c}73.8256 \\
0\end{array}$ \\
\hline 7 & $\begin{array}{c}0.11296 \\
0\end{array}$ & 7.642029 & $\begin{array}{c}1.03157 \\
1\end{array}$ & $\begin{array}{c}20.9737 \\
1\end{array}$ & $\begin{array}{c}70.3526 \\
9\end{array}$ \\
\hline 8 & $\begin{array}{c}0.11917 \\
4\end{array}$ & 8.780516 & $\begin{array}{c}1.16723 \\
4\end{array}$ & $\begin{array}{c}22.3806 \\
1\end{array}$ & $\begin{array}{c}67.6716 \\
4\end{array}$ \\
\hline 9 & $\begin{array}{c}0.12466 \\
3\end{array}$ & 9.732168 & $\begin{array}{c}1.26548 \\
9\end{array}$ & $\begin{array}{c}23.4759 \\
6\end{array}$ & $\begin{array}{c}65.5263 \\
9\end{array}$ \\
\hline 10 & $\begin{array}{c}0.12956 \\
4\end{array}$ & 10.53105 & $\begin{array}{c}1.33976 \\
4\end{array}$ & $\begin{array}{c}24.3910 \\
7\end{array}$ & $\begin{array}{c}63.7381 \\
1\end{array}$ \\
\hline
\end{tabular}

Fuente: Elaboración propia en base a datos del Banco Central del Ecuador 